\title{
Identité... Décor... Fond... Forme... Ainsi fond, fond, fond...
}

\section{Thibault Fourrier}

\section{OpenEdition}

1 Journals

Édition électronique

URL : https://journals.openedition.org/rbnu/3118

DOI : 10.4000/rbnu.3118

ISSN : 2679-6104

Éditeur

Bibliothèque nationale et universitaire de Strasbourg

Édition imprimée

Date de publication : 1 mai 2010

Pagination : 48-55

ISSN : 2109-2761

\section{Référence électronique}

Thibault Fourrier, «Identité... Décor... Fond... Forme... Ainsi fond, fond, fond... », La Revue de la BNU [En ligne], 1 | 2010, mis en ligne le 01 janvier 2021, consulté le 12 juin 2021. URL : http:// journals.openedition.org/rbnu/3118; DOI : https://doi.org/10.4000/rbnu.3118

\section{(c) (i) (2)}

La Revue de la BNU est mise à disposition selon les termes de la Licence Creative Commons Attribution - Pas d'Utilisation Commerciale - Partage dans les Mêmes Conditions 4.0 International. 
ming

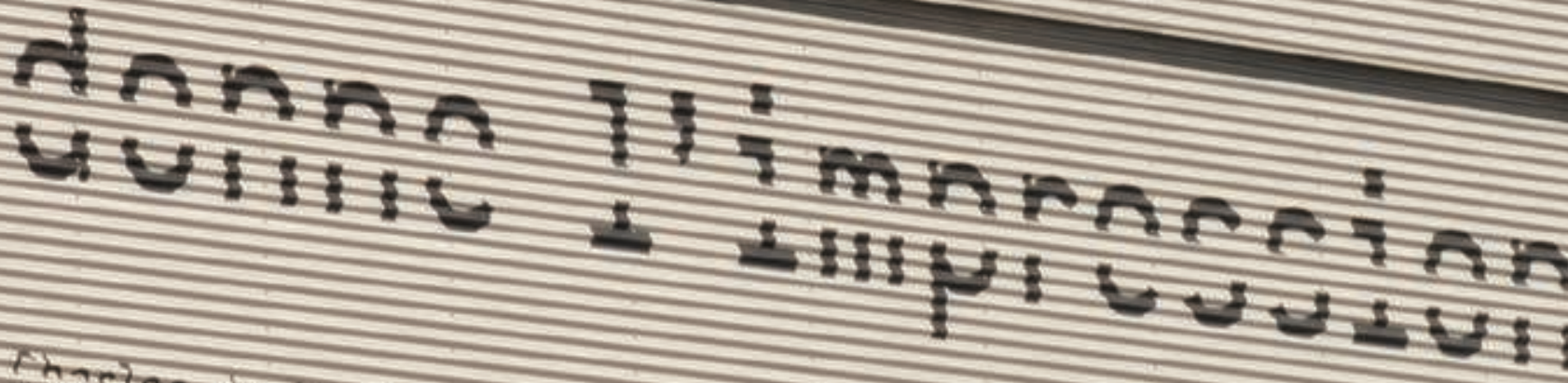
.

in médiatheque

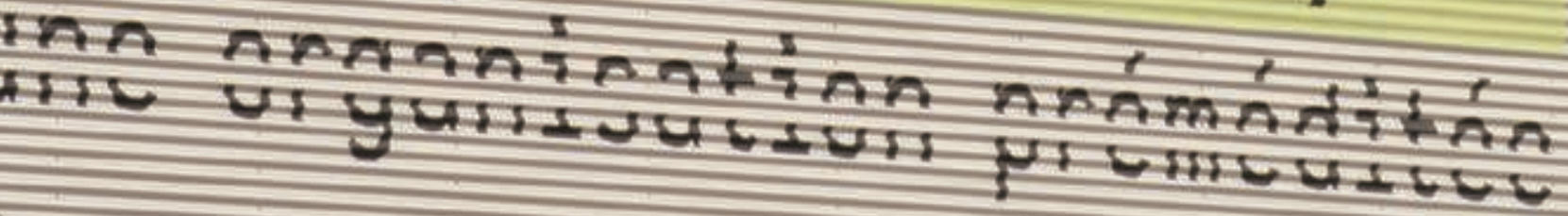




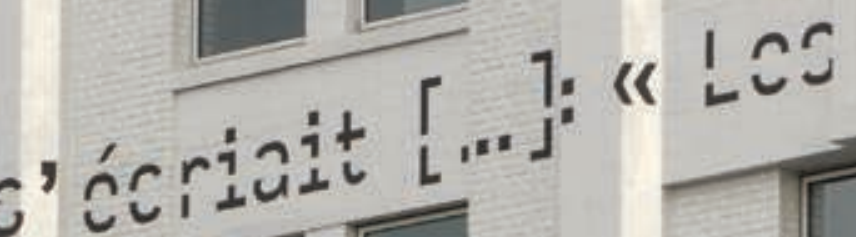

nuivis

trie fonction ist $\mathrm{de}$ ics iutic conitiut
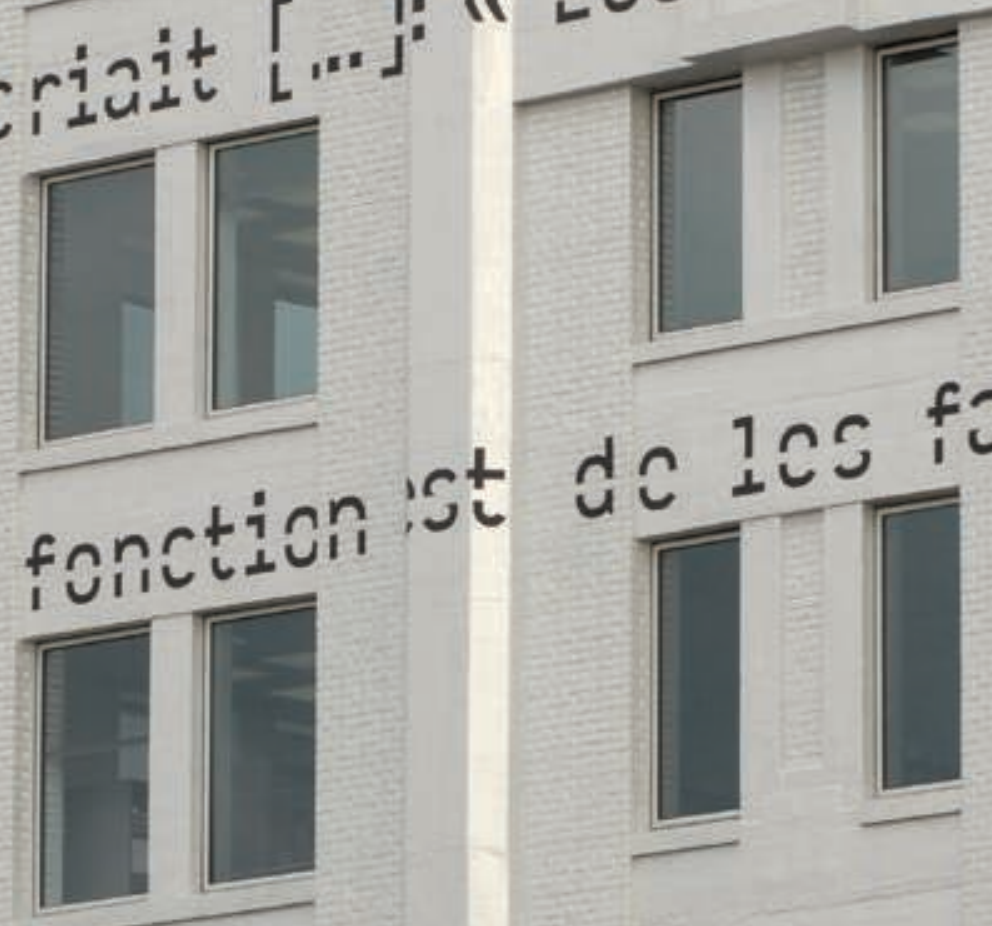

in int ics nossćci"

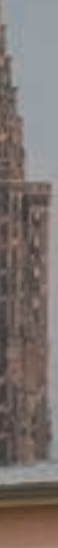

¿u med.athe

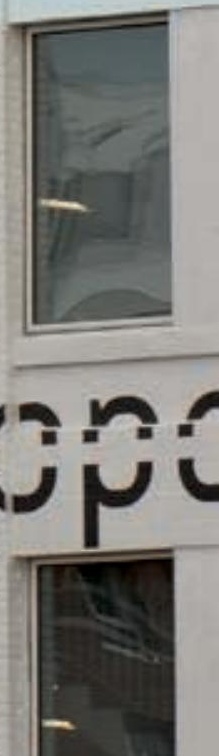




\section{Identité...}

\section{Décor...}

\section{Fond...}

\section{Forme...}

\section{Ainsi fond, fond, fond...}

A

vant tout développement, je souhaite dire combien le travail de recherche bibliographique pour la médiathèque de Strasbourg a été exaltant, riche de découvertes, humainement prenant pour les échanges qu'il a générés, intellectuellement perturbateur et finalement source d'humilité. Je tiens à remercier le commanditaire, Robert Grossmann et toute l'équipe administrative dont les bibliothécaires, pour leur ouverture d'esprit et la marge de manœuvre qu'ils nous ont accordée; les architectes Marc Ibos et Myrto Vitart pour avoir accepté avec générosité et intelligence l'idée que la signalétique vienne tardivement se lover au sein de leur œuvre pour en devenir un élément supplémentaire et non une intrusion; enfin Agathe Bischoff-Morales sans qui je n'aurais jamais pu avoir accès à certaines sources propres aux alsatiques, à l'histoire du site mais également pour une juste perception et une meilleure compréhension de ce qu'est le vif esprit alsacien et plus largement rhénan... Aussi pour le très beau texte qu'elle a fait, aujourd'hui " en miroir " de la salle du patrimoine. Avec enthousiasme et disponibilité, elle a été mon cicérone, mon mentor, ma boussole dans les entrelacs de cette culture aux accents forts et subtils qui m'était étrangère, moi qui ne parle pas un mot d'allemand et encore moins d'alsacien... Grâce à elle j'ai découvert Claude Riehl et les frères Matthis entre autres (" spéciale dédicace " à Mireille Petry) et en ris encore ! J'espère ne pas molester, par ces mots, sa naturelle modestie. Je remercie bien sûr Ruedi de m'avoir appelé sur ce projet, continuant ainsi une collaboration nourricière, joyeuse et exigeante amorcée il y a plus d'une quinzaine d'années.

Harassant par sa durée et l'attention sans faille qu'il requérait, ce projet fut un réel plaisir et je dois reconnaître, avec une pointe d'orgueil, que le résultat me plaît infiniment, que je suis fier (toute humilité tue), en ma qualité de cheville ouvrière appartenant à un tout, d'y avoir contribué.

$\mathrm{Au}$ début de cet article a été posée, entre autres, la question de l'aspect décoratif émanant de l'architecture. Une fois sorti du théâtre, de l'élégance émotive des napperons de dentelle et draps plus que centenaires, du charme équivoque ou fascinant de certains intérieurs, je n'éprouve pas de sympathie particulière pour les termes " décor » ou " décoration ". Ils m'évoquent plus un masque ou un artifice provisoire, une excroissance semblant naturelle, superfétatoire ou neutre par absence de sens profond, un " truc "... en tout cas, rien qui soit en rapport avec " la litho ", celle de l'âge grec, solide et minérale (mais en résonance aussi avec la lithographie du XIX ${ }^{e}$ siècle dans le cas d'une médiathèque), qui entretient un lien privilégié avec le temps, la durée. Le bâti traverse le temps. Le décor ne s'inscrit pas, dans mon esprit, dans cette 
temporalité. A tout prendre, je crois préférer les mots plus académiques " ornement-ornementation "... Mais jouons le jeu de l'exercice de style avec " décor "; une manière comme une autre d'ornementer le déroulement du texte. En faire un élément princeps du discursif, sachant que ce mot est à l'origine même du raisonnement qui suit, la " première pierre "... Et plus qu'un élément de décor, ce jeu sur les mots pose directement la question ontologique qui doit animer toute réflexion relative à l'élaboration d'une identité, d'un système de représentation ou d'information : partir de l'existant dans toute sa réalité physique et symbolique, exprimer l'être et non le paraître, toujours s'appuyer sur le réel intérieur sans le dévoyer, pour mieux s'en échapper ensuite dans les différentes manifestations publiques mais en conservant le sens de l'origine. Le décor sera donc le point de départ et le fil conducteur de cette courte réflexion.

Le premier point d'argumentation est de préciser que si le projet signalétique participe à l'aspect décoratif de l'architecture de la Médiathèque André Malraux, c'est essentiellement parce qu'il est intégré dans cette même architecture, qu'il s'exprime dans la matière même des murs et des sols, qu'il en épouse les formes et les contraintes. La signalétique devient en quelque sorte une seconde peau, un greffon bavard ayant pour fonction d'orienter tout en étant le miroir de la fonction même du lieu (dispenser généreusement du verbe et de la pensée), mais un miroir déformant, car elle le fait de manière ludique, partielle et tronquée, sans ambition de délivrer de la connaissance, tout au plus de solliciter la curiosité. Miroir déformant donc, dans le sens où il s'éloigne de la fonction du lieu car se limitant, formellement, à être l'évocation de la mission et du contenu, sans autre prétention.

Le deuxième point quant à la dimension décorative tient au fait que le programme signalétique utilise les partis pris architecturaux (traitements des volumes, répartition et formes des à-plats de couleurs rouge/ gris, angles...) et de design environnemental (mobilier et accessoires comme le miroir) comme support à sa présence, se les approprie mais sans modifier l'existant sur le fond, uniquement en les habillant, en les habitant. Si l'on fait disparaître les éléments signalétiques, l'aspect décoratif entre autres par la colorimétrie ou la mise en scène d'éléments fonctionnels (tuyauterie de ventilation, piliers, mobilier) reste et se suffit à lui-même. Donc le geste architectural porte déjà en son sein une potentialité " décorative " que le système signalétique ne fait qu'appuyer, magnifier, extrapoler et d'une certaine manière phagocyter mais sans le pervertir. D'une certaine manière, il fait " sortir les murs des murs ", légitimé en cela par sa fonction utilitaire d'orientation. Pour autant, indépendamment, les choix architecturaux vont bien au delà d'un décor. Ils définissent une vie nouvelle, affirment une personnalité dans son originalité tout en assimilant la mémoire du lieu pour la faire renaitre afin de créer les conditions d'une nouvelle histoire qui passe outre son passé sans le négliger ou l'oublier. Le temps est donc omniprésent, le curseur glisse du passé au futur, simplement. Et la signalétique suit le mouvement.

Si l'on se laisse aller à " capillotracter " le raisonnement, on pourrait "pulsoboissuner" (ou "pulsobouchonner ") plus loin en affirmant que la signalétique applique cette logique de l'intérieur : les écrits sont aussi bien anciens que contemporains et quant au futur, il s'opère dans la capacité du système, prévue, à accueillir de nouveaux apports littéraires. Ainsi, la contemporanéité peut aussi perdurer. Lieu commun : le temps est mouvement fait d'arrêts sur image continus et concordants, parfois non, en abîme. Toute la problématique est de donner vie à un système identitaire afin qu'il accompagne naturellement, intrinsèquement, en évidence, l'évolution de "l'être ", en l'occurrence un lieu de vie collective ouvert diffusant la connaissance au sens le plus exhaustif, " babélien "... En aucun cas une chose figée. Soyons clair, le système ne donne pas vie à l'expression du lieu. Il est la manifestation visible d'une personnalité vivante en mouvement, pas un masque, pas la " persona " étrusque. Décorer devient anecdotique. [Aparté : je pense à Borges et Virilio en écrivant. Borges est présent dans la médiathèque, pas Virilio... La seule bribe de regret que je pourrais nourrir serait éventuellement tous ces chers absents. Mais je préferre avoir à l'esprit tous les chers présents et puis... l'incomplétude était de toute façon comprise au début de l'exercice.] 


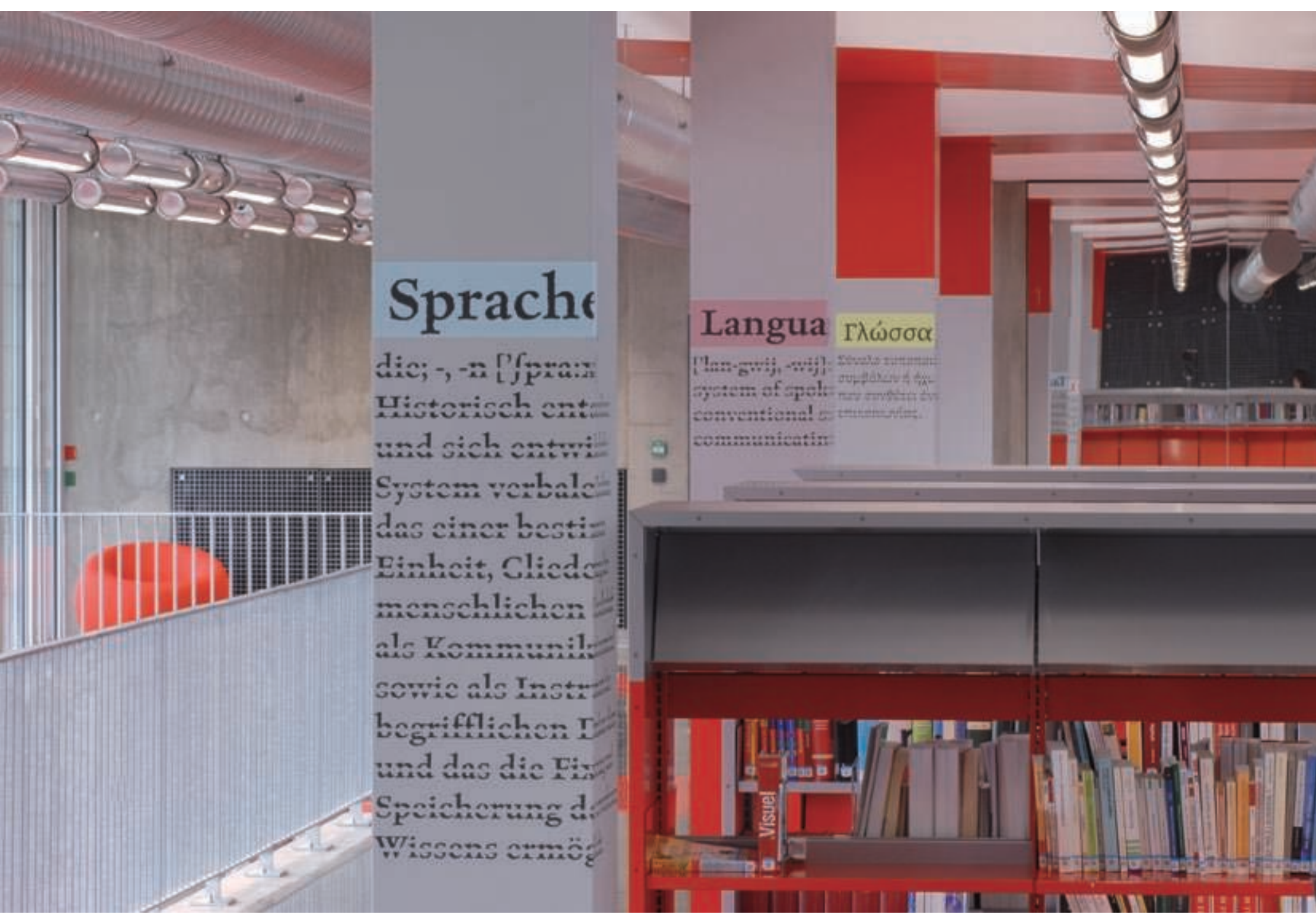

\ Médiathèque Malraux, signalétique interne 
Le caractère décoratif que l'on peut attribuer à ce système signalétique est donc ambigu, puisqu'il est à la fois un élément rapporté mais sans être un objet physique rajouté, un ornement fonctionnel comme le sont souvent les supports signalétiques (premier paradoxe). Ensuite, tout en répondant à une fonction bien précise (orienter/informer), il ne fait que suivre les lignes directrices tracées par l'architecture ellemême avec l'ambition non de la décorer, mais bien de "disparaître en elle-apparaître à travers elle", d'en être une émanation naturelle dévoyée (deuxième paradoxe).

Si je voulais tenter un parallèle hasardeux, j'évoquerais le cas des hiéroglyphes... Intégrés dans la masse et les méandres des pyramides, mêmes s'ils peuvent être appréhendés aujourd'hui (avec un brin de désinvolture, quand même) comme "décoratifs", ils avaient manifestement à leur époque une fonction votive, cérémonielle, honorifique, confessionnelle ou informative pour les infortunés premiers touristes... De là à dire ou penser que la signalétique pourrait honorer ou glorifier un dieu vivant comme Pharaon, c'est gonflé ! Cependant, dans un cadre polythéiste à caractère terrestre et républicain parfaitement arbitraire, on peut cavalièrement considérer la littérature comme une manne à respecter, préserver, invoquer, chercher, écouter, voire vénérer avec modération pour espérer être sauvé de l'obscurité, ou plus justement de l'obscurantisme. La médiathèque devient temple et moi, je m'égare...

Par contre, là où une valeur décorative et esthétique affectée peut être attribuée clairement au système signalétique, c'est dans le choix des différentes typographies pour chaque département, le " trait-rature " estompant le texte et les cartouches de couleurs permettant de mettre en exergue les signifiants. Car nous sommes bien là dans une logique formelle, une écriture plastique où l'aspect l'emporte sur le sens (et encore, ce postulat n'est pas si simpliste puisque chaque "famille " typographique fait référence à la nature du département qu'elle identifie...). Pour autant, si le traitement graphique a une vertu décorative, il a aussi une fonction : le "trait-rature " homogénéise les textes et les hiérarchise comme secondaires dans la logique d'information, alors que le cartouche de couleur indique le premier niveau de lecture à l'usager, l'impératif. La signalétique a d'abord une valeur d'usage, elle fonctionne, elle sert. Si l'imaginaire et l'esthétisme sont des vertus reconnues au design, sa justification première est de répondre à un besoin ou une fonction, d'être utile avant tout, avec agrément. Et il peut aussi produire du sens, en dehors de son utilité propre, son sens premier.

Finalement, la signalétique n'a pas pour vocation d'être décorative. Si elle l'est, c'est essentiellement parce qu'elle est interprétée comme telle du fait qu'elle se substitue par endroits à la seule architecture et qu'elle entretient une exigence esthétique, plastique. Pour reprendre le thème même de l'article qui comporte le terme "identité ", je serais tenté de dire que la signalétique, dans beaucoup de cas mais particulièrement dans celui de la Médiathèque André Malraux, à Strasbourg, a plus pour fonction simultanée (après orienter/informer, sa nécessité) de personnifier, singulariser, participer à l'affirmation d'une identité propre et unique, imposer en douceur et dans le temps par imprégnation progressive dans le décor une originalité tranquille et campée, sans effets disproportionnés, de fait.

Si l'on observe de loin la médiathèque dans son paysage immédiat, on voit bien que sa personnalité s'impose d'abord par le bâti (une présence), et qu'ensuite elle revendique son unicité identitaire entre autres par sa signalétique murale (chose qui sera d'autant plus sensible quand l'ensemble du site sera aménagé). Alors décor, pas décor ? On peut éventuellement considérer que la Médiathèque André Malraux décore le paysage, mais ce dont on peut être sûr, c'est qu'elle l'habite, s'y affirme, s'y enracine. Et de l'arbre sort un fruit, le livre... On n'en sort pas !

\section{Thibault Fourrier}




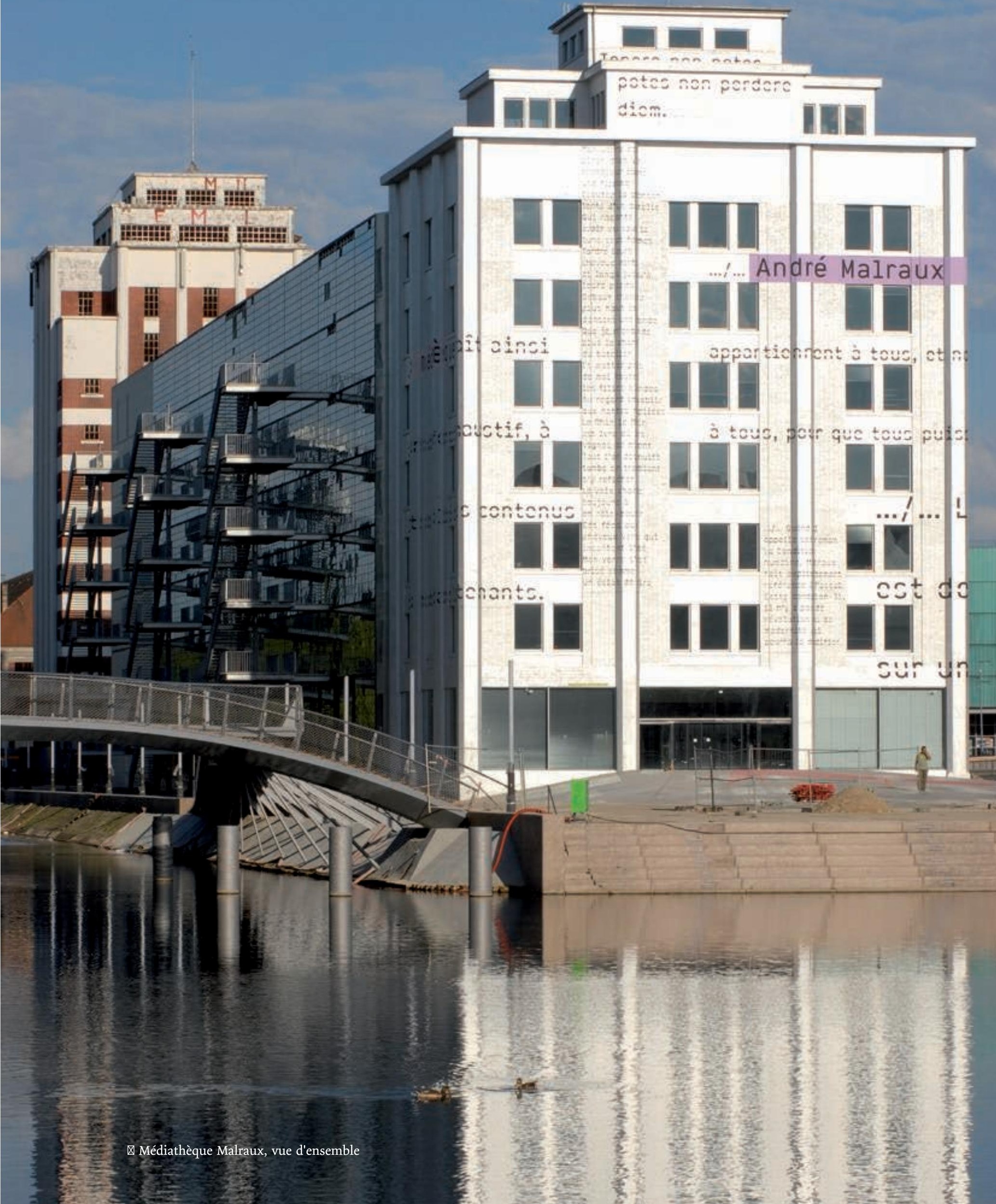

